PRATISSOLI, D.; PEREIRA, F.F.; BARROS, R.; PARRA, J.R.P.; PEREIRA, C.L.T. Parasitismo de Trichogramma pretiosum em ovos da traça-das-crucíferas sob diferentes temperaturas. Horticultura Brasileira, Brasília, v.22, n.4, p.754-757, out-dez 2004.

\title{
Parasitismo de Trichogramma pretiosum em ovos da traça-das-crucíferas sob diferentes temperaturas
}

\author{
Dirceu Pratissoli ${ }^{1}$; Fabricio F. Pereira ${ }^{1}$; Reginaldo Barros² ${ }^{2}$ José R.P. Parra ${ }^{3}$; Cácia L.T. Pereira ${ }^{1}$ \\ ${ }^{1}$ UFES, Alto Universitário S/N, C. Postal 16, 29500-000 Alegre-ES; ${ }^{2}$ Depto. Agronomia/Fitossanidade, UFRPE, Av. Dom Manuel de \\ Medeiros S/N, Dois Irmãos, 52171-900 Recife-PE; ${ }^{3}$ Depto. Entomologia, Fitopatologia e Zoologia Agrícola, ESALQ/USP, C. Postal 09, \\ 13418-900 Piracicaba-SP; E-mail: ffpereira@insecta.ufv.br; dirceu@npd.ufes.br; cacia@bol.com.br; jrpparra@carpa.ciagri.usp.br; \\ rbarros@ufrpe.br
}

\section{RESUMO}

Estudou-se a capacidade de parasitismo de Trichogramma pretiosum Riley (Hymenoptera: Trichogrammatidae), em ovos de Plutella xylostella (L.)(Leptoptera: Plutellidae) nas temperaturas de 18; 20;22; $25 ; 28 ; 30$ e $32^{\circ} \mathrm{C}$, avaliando-se número de ovos parasitados diariamente, porcentagem acumulada de parasitismo, número total de ovos parasitados por fêmea e longevidade de fêmeas. $\mathrm{O}$ ritmo de parasitismo durante as primeiras 24 horas, oscilou de 1,6 a 16 ovos parasitados por fêmea de T. pretiosum nas temperaturas entre 18 e $32^{\circ} \mathrm{C}$. O parasitismo acumulado de ovos de P. xylostella, nas temperaturas de $18 ; 20 ; 22 ; 25$; $28 ; 30 \mathrm{e} 32^{\circ} \mathrm{C}$, atingiu $80 \%$, aos $2 ; 16 ; 11 ; 3 ; 5 ; 4 \mathrm{e} 7$ dias, por T. pretiosum. As maiores taxas de parasitismo ocorreram nas faixas térmicas de 25 ; 28 e $30^{\circ} \mathrm{C}$. A longevidade média de fêmeas de $T$. pretiosum nas faixas térmicas compreendidas entre 18 e $32^{\circ} \mathrm{C}$, variaram de 2,3 a 13,3 dias.

Palavras-chave: Plutella xylostella, controle biológico, Trichogrammatidae.

\begin{abstract}
Parasitism of Trichogramma pretiosum on diamondback moth eggs under different temperatures

The parasitism capacity of Trichogramma pretiosum Riley (Hymenoptera: Trichogrammatidae) on eggs of Plutella xylostella (L.) (Lepidoptera: Plutellidae) was studied, under temperatures of 18; 20; $22 ; 25 ; 28 ; 30$ and $32^{\circ} \mathrm{C}$ aiming to evaluate the number of days with parasitism, cumulated parasitism, total number of eggs parasitized per female and their longevity. Parasitism during the first 24 hours ranged from 1.6 to 16 eggs of $P$. xylostella per T. pretiosum female in the range of 18 to $32^{\circ} \mathrm{C}$. Cumulated egg parasitism of $P$. xylostella by $T$. pretiosum reached $80 \%$ after $2 ; 16 ; 11 ; 3 ; 5 ; 4$ and 7 days at $18 ; 20 ; 22$; $25 ; 28 ; 30$ and $32^{\circ} \mathrm{C}$. Higher parasitism rates were recorded at $25 ; 28$ and $30^{\circ} \mathrm{C}$ while longevity of $T$. pretiosum females varied from 2.3 to 13.3 days under temperatures of 18 to $32^{\circ} \mathrm{C}$.
\end{abstract}

Keywords: Plutella xylostella, biological control, Trichogrammatidae.

(Recebido para publicação em 3 de março de 2004 e aceito em 27 de outubro de 2004)

$\mathrm{A}$ tualmente, a traça-das-crucíferas Plutella xylostella (L.) é considerada a praga mais importante de crucíferas em todo o mundo e o custo com seu manejo tem sido estimado em mais de um bilhão de dólares por ano (Talekar e Shelton, 1993). As diversas estratégias de controle para esta praga têm resultado numa acentuada dependência de inseticidas, o que vem propiciando resistência de $P$. xylostella a pesticidas químicos e até mesmo biológicos como os produzidos a partir de Bacillus thuringiensis Berliner (Perez et al.,1997).

O controle biológico de $P$. xylostella quando bem implantado pode ser excelente alternativa frente às habituais recomendações de controle químico (Krnjajic et al.,1997). O elevado número de trabalhos mencionando o complexo de parasitóides nas diferentes regiões produtoras de crucíferas demonstra a importância destes inimigos naturais para manutenção do nível populacional desta praga abaixo do nível de dano econômico (Mitchel et al.,1998).

Dentre estes agentes de controle biológico, os parasitóides de ovos pertencentes ao gênero Trichogramma destacam-se pelo fato de ter ampla distribuição geográfica, serem altamente especializados, além da comprovada eficiência no controle de pragas, sobretudo aquelas pertencentes à ordem Lepidoptera (Zucchi e Monteiro, 1997).

Várias espécies de Trichogramma têm sido mencionadas como eficientes em relação ao seu potencial de uso no controle de $P$. xylostella em diversos países, como T. ostriniae Pang \& Chen, T. chilonis Ishii e T. pintoi Voegelé na Alemanha (Wuhrer e Hassam, 1993), T. pretiosum e T. minutum Riley nos EUA (Vasquez et al., 1997), T. evanescens Westwood na Yugoslavia (Krnjajic et al.,1997); T. voegelei Pintureau, T. oleae Voegelé \& Pointel, T. dendrolimi Matsumura, T. exiguum Pinto \& Platner, T. chilonis, T. pretiosum, T. buesi
Voegelé, T. ostriniae, e Trichogrammatoidea bactrae Nagaraja na França (Tabone et al.,1999).

Aspectos biológicos de T. pretiosum já foram pesquisados em diferentes hospedeiros e temperaturas por Basso et al. (1998) no Chile, Harrison et al. (1985) nos EUA e Wuhrer e Hassan (1993) na Alemanha. Mas no Brasil, à exceção de Barros e Vendramin (1999), são escassos os relatos de pesquisas mencionando aspectos biológicos desse parasitóide quando criado em ovos de P. xylostella.

Um dos fatores que pode ser responsável pelo sucesso ou fracasso da utilização de parasitóides do gênero Trichogramma no controle de lepidópteros-praga é o conhecimento de parâmetros biológicos deste parasitóide quando associado a determinado hospedeiro alvo, tais como capacidade e viabilidade do parasitismo, duração do ciclo de desenvolvimento, razão sexual e longevidade (Fuentes, 1994). Noldus 
(1989) ainda enfatiza que tais características podem ser altamente influenciadas por fatores físicos, como umidade, luz e principalmente temperatura.

Assim, o estudo da capacidade de parasitismo de T. pretiosum em função da temperatura pode fornecer informações importantes para a implantação de programas de manejo integrado de $P$. xylostella, visto que, cada espécie de Trichogramma possui comportamento diferenciado e que pode variar de acordo com suas características intrínsecas, proporcionando uma maior ou menor adequação a um determinado ambiente e hospedeiro (Bleicher e Parra, 1990). Deste modo, esta pesquisa teve como objetivo obter informações básicas sobre aspectos biológicos de T. pretiosum criado em ovos de P. xylostella em diferentes temperaturas.

\section{MATERIAL E MÉTODOS}

\section{Criação de $P$. xylostella}

Inicialmente, cerca de 500 pupas de P. xylostella provenientes da criação estoque do laboratório de Biologia de Insetos da Área de Fitossanidade da (UFRPE), foram transferidas para o laboratório de Entomologia do (CCAUFES), onde foi iniciada a criação da traça-das-crucíferas em folhas de couve, segundo método adotado por Barros e Vendramin (1999).

Coleta, manutenção e multiplicação dos parasitóides

A espécie T. pretiosum, proveniente da criação estoque do laboratório de Entomologia do (CCA-UFES), foi inicialmente coletada em plantios comerciais de tomate localizados no município de Alegre (ES). Para coleta dessa espécie, foram utilizadas cartelas contendo ovos de Anagasta kuehniella (Zeller) inseridas em pequenas gaiolas fixas, por 48 horas, às folhas do terço superior de plantas de tomate. A espécie foi identificada pelo professor R.A. Zucchi, do Depto. de Entomologia da ESALQ em Piracicaba.

Para manutenção dos parasitóides, ovos de A. kuehniella obtidos segundo técnica adaptada de Parra (1997), foram inviabilizados e fixados em retângulos de cartolina azul celeste $(8,0 \times 2,0 \mathrm{~cm})$, usando goma arábica diluída a $30 \%$.
Estas cartelas foram inseridas em tubos de vidro $(8,5 \times 2,4 \mathrm{~cm})$, contendo adultos recém emergidos.

Capacidade de parasitismo de $\boldsymbol{T}$. pretiosum em ovos da traça-dascrucíferas em diferentes temperaturas

Inicialmente ovos de P. xylostella com 12 horas de idade foram coletados de discos de folha de couve e transferidos com auxílio de um pincel de pêlos finos umedecidos para cartelas de cartolina azul celeste $(2,5 \times 0,3 \mathrm{~cm})$. Para cada temperatura $(18 ; 20 ; 22 ; 25 ; 28$; 30 e $32^{\circ} \mathrm{C}$ ), foram isoladas 20 fêmeas recém emergidas, em tubos de vidro (3,0 x $0,4 \mathrm{~cm})$, fechados com filme plástico PVC, contendo no seu interior gotículas de mel para alimentação dos adultos. Cartelas contendo 30 ovos do hospedeiro, quantidade esta ajustada em função de testes preliminares, foram diariamente introduzidas nos tubos para cada fêmea individualizada. A troca das cartelas foi realizada diariamente, sempre a partir das 15 horas. As cartelas com ovos parasitados no dia anterior foram transferidas para sacos plásticos $(23 \mathrm{x}$ $4,0 \mathrm{~cm}$ ), fechados, e mantidos em câmaras climatizadas reguladas para as temperaturas citadas anteriormente, com umidade relativa de $70 \pm 10 \%$ e fotofase de 14 horas até a emergência dos descendentes. Foram avaliados os parâmetros biológicos: ritmo de parasitismo diário; porcentagem acumulada de parasitismo; número total de ovos parasitados por fêmea e longevidade das fêmeas.

$\mathrm{O}$ delineamento experimental utilizado foi inteiramente casualizado, com vinte repetições. Os dados foram submetidos à análise de variância e as médias comparadas pelo teste de Tukey $(\mathrm{Pd} \leq 0,05)$, utilizando-se o programa computacional SANEST (Versão 3.0).

\section{RESULTADOS E DISCUSSÃO}

$\mathrm{O}$ ritmo de parasitismo durante as primeiras 24 horas, oscilou de 1,6 a 16 ovos parasitados por fêmea de $T$. pretiosum nas temperaturas entre $18 \mathrm{e}$ $32^{\circ} \mathrm{C}$ (Fig. 1). Os maiores números de ovos parasitados foram 16; 12 e 14 ovos no primeiro dia, nas temperaturas de 25 ; 28 e $30^{\circ} \mathrm{C}$, respectivamente, com maior concentração de postura nos primeiros dias (Fig. 1). Fenômeno semelhante foi constatado por Tironi (1992) para duas espécies de Trichogramma, embora este pesquisador tenha relatado que os parasitóides mantiveram o parasitismo até a morte, fato que não aconteceu nesta pesquisa. Resende e Ciociolla (1996) obtiveram resultados em que o período de parasitismo em ovos de Helicoverpa zea Boddie por T. atopovirilia Oatman \& Platner variou de 4 a 12 dias, com maior concentração de postura nas primeiras 24 horas, nas temperaturas de 15 ; $20 ; 25$ e $30^{\circ} \mathrm{C}$.

As variações de parasitismo observadas, bem como as relatadas por outros autores, podem estar relacionadas, segundo Noldus (1989), à técnica de criação utilizada, ao hospedeiro natural do parasitóide, à procedência da linhagem, à geração de laboratório, às condições de temperatura, umidade relativa, e ao fotoperíodo e hospedeiro alternativo utilizado para manutenção das criações de laboratório.

As fêmeas de T. pretiosum diminuíram o número de ovos colocados diariamente em função do tempo de parasitismo nas condições térmicas em que foram submetidas, podendo-se, desta forma, inferir que o parasitismo tende a decrescer com o passar do tempo. Fato similar também foi constatado para outras espécies de Trichogramma, sobre hospedeiros diferentes (Parra e Sales Jr., 1995; Sá e Parra, 1994; Resende e Ciociolla, 1996; Inoue e Parra, 1998).

O parasitismo acumulado de ovos de P. xylostella, nas temperaturas de 18; 20; 22; 25; 28; 30 e $32^{\circ} \mathrm{C}$ atingiu $80 \%$ do total de ovos parasitados para cada faixa térmica, aos 2; 16; 11; 3; 5; 4 e 7 dias (Fig. 1). Pratissoli e Parra (2001) atribuiram, como causa da variação no parasitismo, o uso de diferentes espécies e/ou linhagens de Trichogramma, assim como o hospedeiro utilizado e condições climáticas. Isto foi observado também por Marston e Ertle (1973), que registraram o decréscimo total do parasitismo de T. minutum em ovos de Sitotroga cerealella (Oliver), após o $23^{\circ}$ dia com poucos ovos sendo parasitados depois do $10^{\circ}$ dia, bem como Inoue (1997) citado por Inoue e Parra (1998), que detectou parasitismo de T. pretiosum em ovos de $S$. cerealella até $13^{\circ}$ dia, 


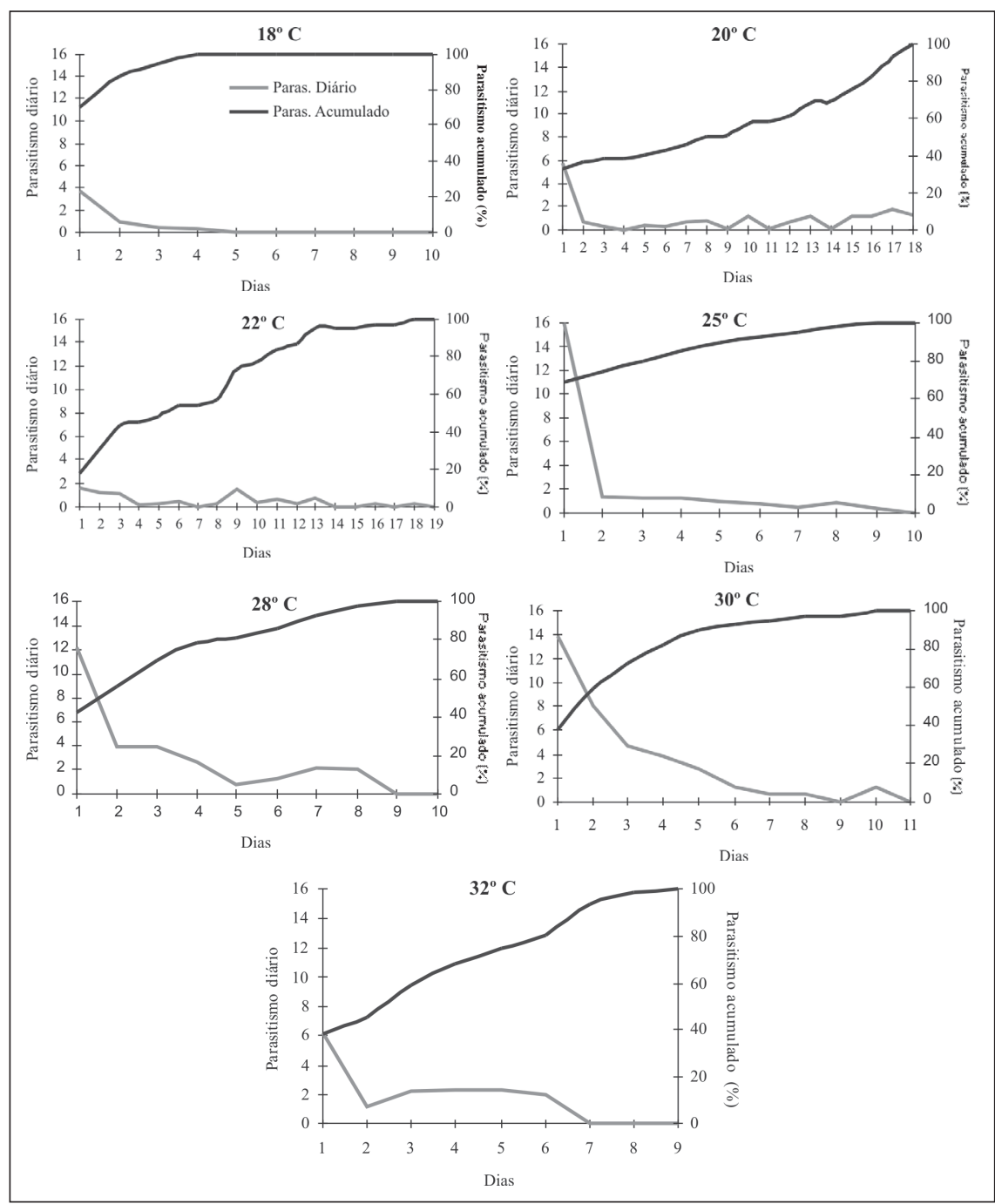

Figura 1. Parasitismo diário e acumulado de Ttichogramma pretiosum mantido em ovos de Prutella xylostella sob sete temperaturas. UR: $70 \pm 10 \%$ e fotofase de 14 horas. As setas indicam 80\% de parasitismo. Alegre (ES), UFES, 2003.

Tabela 1. Médias $( \pm)^{1}$ do número total de ovos parasitados por fêmea e longevidade de Trichogramma pretiosum sobre Plutella xylostella, sob sete temperaturas. UR: $70 \pm 10 \%$ e fotofase: 14 horas $(n=20)$. Alegre (ES), UFES, 2003.

\begin{tabular}{ccc}
\hline Temperatura $\left({ }^{\circ} \mathrm{C}\right)$ & $\begin{array}{c}\text { Total de ovos } \\
\text { parasitados/fêmea }\end{array}$ & Longevidade (dias) \\
\hline 18 & $5,1 \pm 1,30 \mathrm{~b}$ & $13,3 \pm 1,69 \mathrm{a}$ \\
20 & $11,4 \pm 2,86 \mathrm{~b}$ & $12,1 \pm 2,67 \mathrm{a}$ \\
22 & $7,1 \pm 3,46 \mathrm{~b}$ & $11,2 \pm 2,53 \mathrm{a}$ \\
25 & $21,6 \pm 2,70 \mathrm{a}$ & $5,9 \pm 1,09 \mathrm{~b}$ \\
28 & $24,9 \pm 5,33 \mathrm{a}$ & $5,9 \pm 0,77 \mathrm{~b}$ \\
30 & $28,2 \pm 6,62 \mathrm{a}$ & $5,8 \pm 1,42 \mathrm{~b}$ \\
32 & $8,8 \pm 3,82 \mathrm{~b}$ & $2,3 \pm 1,09 \mathrm{c}$ \\
\hline
\end{tabular}

${ }^{1}$ Médias seguidas de mesma letra minúsculas, na coluna, não diferem entre si, pelo teste de Tukey, ao nível de 5\% de probabilidade. Intervalo de confiança (IC).

atingindo $80 \%$ deste parasitismo por volta do $7^{\circ}$ dia e Sá e Parra (1994), que ao estudarem diferentes linhagens de T. pretiosum sobre ovos de Ephestia kuehniella (Zeller) observaram que, de maneira geral, o parasitismo foi maior nos primeiros quatro dias de vida da fêmea.
O número total de ovos de $P$. xylostella parasitados por $T$. pretiosum foi maior na temperatura de $30^{\circ} \mathrm{C}$, embora sem diferir de 28 e $25^{\circ} \mathrm{C}$ (Tabela 1). As menores taxas de parasitismo de T. pretiosum ocorreram nas temperaturas de $18 ; 20 ; 22$ e $32^{\circ} \mathrm{C}$ sendo, portanto, estas faixas térmicas menos adequadas para essa espécie.

Apesar da capacidade de parasitismo de espécies de Trichogramma serem influenciadas pela temperatura e hospedeiros utilizados, Harrison et al., (1985) observaram resultados semelhantes ao deste trabalho; as maiores taxas de parasitismo em ovos de Heliothis virescens Fabricius para T. pretiosum $\mathrm{e}$ T. exiguum também se encontravam a 25 e $30^{\circ} \mathrm{C}$. Tendência similar foi atestada por Navarro e Marcano (1997), ao citarem 23 e $28^{\circ} \mathrm{C}$ como as temperaturas que proporcionaram o maior número de ovos de H. zea parasitados por $T$. pretiosum e T. caiaposi (Brum, Moraes e Soares), respectivamente.

A longevidade média de fêmeas de T. pretiosum, em ovos de P. xylostella apresentou uma relação inversa com o aumento da temperatura (Tabela 1). Pôde-se verificar longevidades semelhantes nas temperaturas de 18; $20 \mathrm{e}$ $22^{\circ} \mathrm{C}$. A partir desta temperatura, esta espécie diminuiu sua longevidade, mas mantendo-se em igualdade, exceto a $32^{\circ} \mathrm{C}$, onde foi constatado o menor período de vida das fêmeas. As maiores longevidades observadas nas temperaturas inferiores podem estar relacionadas a uma diminuição da atividade metabólica em função da diminuição da temperatura, fenômeno este relatado por Bleicher e Parra (1989).

A longevidade de Trichogramma em temperaturas distintas pode apresentar valores diferentes, de acordo com o hospedeiro e a espécie e/ou linhagem deste parasitóide (Parra e Sales Jr., 1995). No entanto, é conveniente salientar que a temperatura, embora seja considerado um fator de extrema importância, não é o único responsável pela alteração no desenvolvimento e longevidade dos insetos, uma vez que outros fatores abióticos como fotoperíodo, umidade relativa e bióticos como competição inter e intraespecífica, podem interferir nas características biológicas de um in- 
seto (Pratissoli e Parra, 2001).

Os resultados obtidos nesta pesquisa evidenciaram que $T$. pretiosum melhor se adaptou a traça-das-crucíferas nas temperaturas de 25 a $30^{\circ} \mathrm{C}$, faixas térmicas onde este parasitóide demonstrou maiores taxas de parasitismo. Assim, conclui-se que $T$. pretiosum têm potencial para utilização em programas de controle biológico de P. xylostella em localidades com estas condições climáticas. Outros estudos relacionados à seleção de linhagens, número ideal e intervalo de liberações dos parasitóides, além da capacidade de dispersão do mesmo, ainda precisam ser feitos para que tais agentes de controle biológico possam se destacar frente aos atuais métodos de supressão desta praga.

\section{AGRADECIMENTOS}

À CAPES, pela concessão da bolsa de estudo ao segundo autor, a UFRPE e ao laboratório de Entomologia do CCA da UFES, por possibilitarem a execução da presente pesquisa.

\section{LITERATURA CITADA}

BARROS, R.;VENDRAMIN, J.D. Efeito de cultivares de repolho, utilizados para criação de Plutella xylostella (L.) (Lepidoptera: Plutellidae), no desenvolvimento de Trichogramma pretiosum Riley (Hymenoptera: Trichogrammatidae). Anais da Sociedade Entomológica do Brasil, Londrina, v.28, n.3, p.469-476, 1999.

BASSO, C.; GRILLE, G.; POMPANON, F.; ALLEMAND, R.; PINTUREAU, B. Comparacion de los caracteres biológicos y etológicos de Trichogramma pretiosum y de $T$. exiguum (Hymenoptera: Trichogrammatidae). Revista Chilena de Entomologia, v.25, n.1, p.4553, 1998.

BLEICHER, E.; PARRA, J.R.P. Espécies de Trichogramma parasitóides de Alabama argillacea. I. Biologia de três populações. Pesquisa Agropecuária Brasileira, Brasília, v.24, n.8, p.929-940, 1989.
BLEICHER, E.; PARRA, J.R.P. Espécies de Trichogramma parasitóides de Alabama argillacea. II. Tabela de vida de fertilidade e parasitismo de três populações. Pesquisa Agropecuária Brasileira, Brasília, v.25, n.2, p.207-214, 1990.

FUENTES, S.F. Producción y uso de Trichogramma como regulador de plagas. Lima, Red de acción em alternativas al uso de agroquímicos (RAAA), 1994.192 p.

HARRISON, W.W.; KING, E.G.; OUZTS, J.D. Development of Trichogramma exiguum and $T$. pretiosum at five temperature regimes. Environmental Entomology, v.14, n.2, p.118-121, 1985.

INOUE, M.S.R.; PARRA, J.R.P. Efeito da temperatura no parasitismo de Trichogramma pretiosum Riley, 1879 sobre ovos de Sitotroga cerealella (Olivier, 1819). Scientia Agricola, Piracicaba, v.55, n.2, p.222-226, 1998.

KRNJAJIC, S.; DIMIC, N.; PERIC, P.; VURSA, M.; CVETKOVIC, M. Biological control of cabbage pests. Acta Horticulturae, v.462, n.1, p.119-124, 1997.

MARSTON, N.; ERTLE, L.R. Host influence on the bionomics of Trichogramma minutum. Annals of the Entomological Society of America, v.66, n.5 p.1155-1162, 1973.

MITCHELL, E.R.; HU, G.Y.; OKINE, J.S Diamondback moth (Lepidoptera: Plutellidae) infestation and parasitism by Diadegma insulare (Hymenoptera: Ichneumonidae) in collards and adjacent cabbage fields. Florida Entomologist, v.80, n.1, p.54-61, 1998

NAVARRO, R.V.; MARCANO, R. Efecto de la temperatura sobre las características biológicas de Trichogramma pretiosum Riley y T. caiaposi (Brun, Moraes y Soares). Agronomia Tropical, v.47, n.3, p.287-297, 1997.

NOLDUS, L.P.J.J. Semiochemicals, foraging behavior and quality of entomophagous insects for biological control. Journal of Applied Entomology, v.108, n.1, p.425-451, 1989.

PARRA, J.R.P.; SALES, JR.O. Biology of Trichogramma galloi Zucchi reared on natural and factitious hosts under different temperatures and relative humidities. Les Colloques de Li'nna, v.73, n.1, p.95-99. 1995.

PARRA, J.R.P. Técnicas de criação de Anagasta kuehniella, hospedeiro alternativo para produção de Trichogramma, In: PARRA, J.R.P.; ZUCCHI, R.A. Trichogramma e o controle aplicado. Piracicaba: FEALQ, cap.12, p.121-150. 1997. PEREZ, C.J.; SHELTON, A.M.; ROUSH, R.T. Managing diamondbackmoth (Lepidoptera: Plutellidae) resitance to foliar applications of Bacillus thuringiensis: testing strategies in field cages. Journal of Economic Entomology, v.90, p.1463-1470, 1997.
PRATISSOLI, D.; PARRA, J.R.P. Seleção de linhagens de Trichogramma pretiosum Riley, 1879, para o controle das traças, Tuta absoluta (Meyrick) e Phthorimaea operculella (Zeller). Neotropical Entomology, Londrina, v.30, n.2, p.277-282, 2001. RESENDE, D.L.M.C.; CIOCIOLLA, A.I. Capacidade de parasitismo de Trichogramma atopovirilia Oatman \& Platner, 1983 (Hymenoptera: Trichogrammatidae) em ovos de Helicoverpa zea (Boddie, 1850) (Lepidoptera: Noctuidae) em diferentes temperaturas. Ciência e Agrotecnologia, Lavras, v.20, n.4, p.421-424, 1996.

SÁ, L.A.N.; PARRA, J.R.P. Biology and parasitism of Trichogramma pretiosum Riley (Hym., Trichogrammatidae) on Ephestia kuenhniella (Zeller) (Lep., Pyralidae) and Heliothis zea (Boddie) (Lep., Noctuidae) egg. Journal of Applied Entomology, Berlin, v.118, n.1, p.38-43, 1994.

TABONE, E.; PINTUREAU, B.; PIZZOL, J.; MICHEL, F.; BARNAY, O.; TABONE, E. Aptitude de 17 souches de Trichogrammes a parasiter lateigne dês cruciferes Plutella xylostella L. em laboratoire (Lep.: Yponomeutidae). Annales de la Société Entomologique de France, v.35, n.1, p.427-433,1999.

TALEKAR, N.S.; SHELTON, A.M. Biology, ecology, and management of the diamondback moth. Annual Review of Entomology, v.38, n.1, p.275-301, 1993.

TIRONI, P. Aspectos bioecológicos de Trichogramma petiosum Riley, $1879 e$ Trichogramma atopovirilia Oatman \& Platner, 1983 (Hym.: Trichogrammatidae), como agentes de controle biológico de Helicoverpa zea (Boddie, 1850) (Lep.: Noctuidae). 1992. 74 p. (Tese mestrado), UFLA, Lavras.

VASQUEZ, L.A.; SHELTON, A.M.; HOFFMANN, M.P.; ROUSH, R.T. Laboratory evaluation of commercial Trichogrammatid products for potential use against Plutella xylostella (L.) (Lepidoptera: Plutellidae). Biological Control, v.9, n.1, p.143 -148, 1997.

WUHRER, B.G.; HASSAN, S.A. Selection of effective species/strains of Trichogramma (Hym., Trichogrammatidae) to control the diamondback moth Plutella xylostella L. (Lep., Plutellidae). Journal of Applied Entomology, v.116, n.1, p.8089, 1993.

ZUCCHI, R.A.; R.C, MONTEIRO. O gênero Trichogramma na América do Sul. In: PARRA, J.R.P.; ZUCCHI, R.A. (eds.), Trichogramma $e o$ controle aplicado. Piracicaba: FEALQ, cap.1, p.41-66. 1997. 